\title{
Upholding parameters of care in a service-provision constrained time period
}

SADJ October 2020, Vol. 75 No. 9 p 474

\author{
LM Sykes
}

Advances in technology necessitate constant changes in all aspects of medical education and patient care. This requires educators and clinicians to merge traditional practices with new innovations, materials and techniques. We are also aware that rapid and dramatic changes are taking place in the world with respect to urbanisation and migration, science and technology and the future of the earth.

Many traditional and dependable diagnostic devices or treatment modalities, such as the familiar stethoscope, are dying after serving us well for years, and will likely be replaced with a handheld ultra-sonic device. With these rapid advances, and the momentum of the Fourth Industrial revolution, the curricula, teaching, learning spaces. examination methodologies, and clinical practices also need to transform. "There is a demand for us to try and solve many of the crucial healthcare challenges through translational research that transverses various disciplines. This will entail integration of Big Data platforms for collection and interpretation of information, as well as practical skills training using state of the art technology". ${ }^{1}$ To this end the profession needs to remain current, and to adapt to the changing world.

In the past, clinicians and educators have generally had the luxury of time, allowing them to make considered and cautious decisions before changing their routine management protocols or embarking on new ventures. The unforeseen COVID-19 pandemic led to widespread disruption in all aspects of medicine and dentistry and necessitated immediate and often drastic changes.

During the last few months we have had to work through a number "teething problems" (excuse the dental analogy) of teaching and service provision under new, uncertain, unexplored and often stressful conditions. Many patients have had to forego routine medical care, visits to doctors and clinics had to be restricted and practitioners have had to make difficult decisions when prioritising needs. It also forced doctors to consider a number of legal and ethical issues and to question their own moral ethos, especially if patients had to be refused treatment. They were faced with the unique situation of having to pro-

Author affiliations:

Leanne M Sykes: BSc, BDS, MDent, Dip Research Ethics (IRENSA); Dip ESMEA (Univ Dundee), DipOdont (Forensic Odontology), Department of Prosthodontics, School of Dentistry, Faculty of Health Sciences, University of Pretoria.

Email: leanne.sykes@up.ac.za vide high quality services in a new and often compromised environment or manner. These demands can be paraphrased well by the parameters set out by Knoernschild ${ }^{2}$ in a position paper on care in prosthodontics. He stated that clinicians must at all times still assess and assure the quality of the care; assist patients and colleagues in clinical decision making; provide education to individuals and in groups via alternative channels; reduce their risks of legal liability as a result of negligent care; help guide the allocation of health resources and identify clinical situations that need to be prioritised or referred to specialist facilities.

At the same time the final decisions must be objective, based on existing scientific evidence, be representative of a professional consensus, and formulated to provide structural flexibility, in order to achieve the desired outcomes.

The education system has also had to adapt. Traditional lectures, ward rounds, and clinical sessions have been replaced with online teaching and learning platforms. Many felt that these changes would compromise the standards of education, and would then impact negatively on future patient care. However, the situation proved to be the exact opposite as it opened up an expansive new world. Teachers had to once again become students and learn how to present their lecture material in different and innovative ways. These included amongst others, use of a variety of internet teaching platforms and chat rooms, development of virtual patients and case scenarios, and even exploration of 3-D technology to fabricate anatomical models that students could use to acquire the manual dexterity and skills needed for the real-life situations. However, perhaps the greatest positive spin off to come from this situation was the explosion of research and publications that the pandemic generated.

Furthermore, this universal crisis seems to have brought people from across the globe together in their pursuit of a vaccine and/or treatment. We can only hope that this unified spirit of collaboration and camaraderie will persist for many years to come, regardless of whatever "new normal" conditions we find ourselves living in.

\section{Reference}

1. Quoted with permission from Prof Tiaan De Jager, Dean of the Faculty of Health Sciences at the University of Pretoria.

2. Knoernschild KL. Parameters of care for the speciality of prosthodontics. J Prosthetic Dentistry. 2020; 29: 3-147. 